\title{
The Role of the Media Identification in Its Health and Quarantine
}

\author{
Chen Zhang ${ }^{1}$
}

${ }^{1}$ Tianjin University of Finance and Economics, Tianjin, China, 300000

\begin{abstract}
KEYWORDS: Media Identification, Health, Quarantine, Role
\end{abstract}
\begin{abstract}
Identification of vectors of infectious diseases is an important part of the frontier health and quarantine work. With the promulgation of China's accession to WTO and the newly revised "International Health Regulations", monitoring and control of vectors of infectious diseases at port more crucial importance. From our inspection and quarantine agencies currently existing monitoring and control mode vectors paper discusses adapt to the new situation, the role of the media in its appraisal of quarantine.
\end{abstract}

\section{Introduction}

Medical vector is a class of creatures serious harm to human health, can spread a variety of diseases. Currently, there are hundreds of human diseases, including infectious diseases and medical vectors 2/3 a direct or indirect relationship. Prevention and control of vector diseases, one by immunization; and on the vector control. No vaccine for the disease vectors, biological control agents is the only feasible precautions.

Medical vector with a wide, large number of widely distributed, adaptability and other characteristics. They have storage, a variety of infectious pathogens spread effect. Take a scientific, accurate and effective methods and measures of biological medicine media monitoring, identification and control, so as to establish a sanitary barrier border crossing to prevent by medical vector-borne diseases frontier port inbound and outbound.

Accurate knowledge of medical vector background population constitutes the frontier port and exotic medical vector species, through the ribs to properly evaluate medical vector-borne diseases frontier port passed in and out of danger, therefore, to accurately identify medical vectors particularly important.

Currently relations, quality control system technology medical vector appraisal of dispersion, the lack of long-term, systematic medical vectors monitoring, identification, reporting, medical vector and disease, international infectious disease epidemiology dynamic information collection, collation analysis, early warning systems. If you can establish remote medical vector identification and early warning system on the basis of analysis of regional medical vectors currently QC system has been set up on the network of biological laboratories, the experts will be able to give full play and use collective wisdom and strength, can greatly improve the quality of this work is to provide the scientific basis for targeted carry out health and quarantine supervision.

\section{The Status and Role of Media Identification in the Health and Quarantine Work}

Frontier health and quarantine departments to fulfill the newly revised "International Health Regulations" international obligations to protect the health and safety of our people present, the global scope of the new emerging infectious disease, nearly 20 years on average each year one or 
more new infectious diseases emerge; past some infectious diseases have been controlled reappeared. WHO Director-General Ms. Brundtland said: "We are on the brink of a global crisis in infectious diseases, no country can avoid suffer, nor what this country can sit back and relax." The regulations no longer against a disease prevention and control, will pass responsibility to prevent "quarantine infectious disease," the outgoing top priority to health and quarantine departments to expand control of all special public health risks and public international concern health emergencies (including biochemical attacks and nuclear radiation). At the same regulations make clear requirements: "to be kept free of infection and contamination status of the port, vehicles, containers, cargo, etc. (including vectors and reservoirs)". These provisions for monitoring and response capacities frontier health and quarantine departments vectors for communicable diseases surveillance and control of biological and port raised higher requirements. Therefore, strengthening the monitoring and control of infectious disease vectors is health and quarantine departments to better fulfill their obligations and the "People's Republic of China Frontier Health and Quarantine Law" to give technical support responsibilities newly revised "International Health Regulations" and important content.

Embodiment TBT measures to guarantee the safety of China's economy with the rapid development of the economy after China joined WTO, foreign technical barriers to take measures to China and increasing frequency, caused huge losses to China's economy. Accordance with the "Sanitary and Phytosanitary Measures (SPS)" the relevant provisions of the WTO, on the entry and exit conveyances, containers, cargo and other objects found in the quarantine medical vectors is an important basis of technical barriers to take measures. Therefore, strengthen and standardize biological vectors for communicable diseases surveillance and control, collect evidence of infectious diseases caused by media States passed, the implementation of measures and technical barriers to trade in a good position in diplomatic negotiations, to maintain our economic security has important role.

Vectors of infectious diseases surveillance and control is a highly technical work, their work directly affects the level of enforcement as law enforcement technique quarantine authority, monitoring and control ability. At present, the port and the local media control belong to the inspection and quarantine agencies and local epidemic prevention departments. Since the port area range division is often not very clear, dead or likely to cause regulatory overlap. Port vectors to perform background investigations also need to refer to the bottom of the media situation in the region. Therefore, to enhance and standardize biological vectors for communicable diseases surveillance and control efforts, not only to build a platform for collaboration and exchange with the local epidemic prevention departments, media to promote Port biological monitoring and research work, but also help improve our overall public health system.

\section{The Situation of Media Identification Work}

Our inspection and quarantine system under quarantine supervision departments at all levels of general health and quarantine supervision will be divided into "quarantine inspection", "Health Authority", "disease surveillance" and "sanitizing" section 4, part 4 serial structure, its core is a quarantine inspection. The "media Biological Monitoring and Control" Work is work in the subsidiary or more specific process several jobs in, not an independent law enforcement and regulatory unit. In addition, the "three seized one", the current health and quarantine inspection and supervision of cargo, container and other quarantine objects or many of the original animal and plant quarantine commodity inspection personnel, and its emphasis on the technical level of 
monitoring and control of vectors relatively low, affecting the further development of vector surveillance and control efforts.

\section{The Problems of Media Identification Work}

Currently, the monitoring and control of biological media is not independent units, and therefore there is no clear division of responsibilities and organizational coordination and management mechanism. Technical support as an important medium for biological monitoring and control efforts, as attached to the health and quarantine inspection and supervision of "mainstreaming", has long been not given due attention, often "thunder and rain" phenomenon, technology personnel and weak phenomenon has not been properly changed. Currently, the port monitoring and control vectors work very hard and are outside the "subject of law enforcement", each inspection and quarantine departments widespread media biological monitoring, identification and detection of cases and a shortage of professionals lean, serious impact on health and quarantine supervision of depth.

\section{The Role of Media Identification in Its Health and Quarantine}

The vector biology health and quarantine supervision is relatively independent, prevent multiple management caused by chaotic management, in favor of vector surveillance and control work flow smooth implementation and data collection for the transport and port unit risk early warning and classification management lay the foundation. Thanks to independent media department of biological regulation, can fully integrate human, material and time, the media transport and port monitoring and port baseline survey work carried out by a department complete from start to finish to ensure the continuity of work and data integrity, ease of analysis and summary, greatly increasing the likelihood of technical barriers to provide evidence for national measures. At the same time, thanks to detailed and complete media information can be aggregated for further analysis, risk analysis according to the severity of the media, the agent of the ship, or hierarchical management, port-based port or other biological media Health Inspection and Quarantine joint prevention and control and provide a favorable basis.

Since the media monitoring is part of the health and quarantine inspection or supervision, in order to ensure that the administrative time license issued by the quarantine certificates, quarantine officers difficult to achieve intensive, generally only through the "visual observation" rough estimate of medium density, easily lead "false negative" to happen. With the new media monitoring and biological control department, we need to resolve this problem. Health and quarantine inspection and quarantine officers of the ship and health supervision process, the media biohazard roughly assess the situation, and fill out the "ship media table." Media sector according to the monitoring results of the inspection and quarantine supervision and basic sanitation conditions, a ship (ship's age, health status of the ship, ship types, from country, season, historical monitoring results, etc.) analysis to determine the need for further media the density of monitoring. Because of staff, drug equipment, time are guaranteed, media monitoring staff can take advantage of "drug shock method" or other devices on the ship for a more detailed method of monitoring density, improve the accuracy of the positive rate and media density. At the same time, the results of biological monitoring density media are the way to take sanitary and quarantine treatment basis. Quarantine officer may determine specific programs sanitary and quarantine treatment according to the degree of hazard medium, and oversee the implementation of sanitary and quarantine treatment and evaluate the effect. 
Evaluation and Research scientific and effective supervision and treatment effect in favor of sanitary and quarantine treatment. The work identified a series of vectors sanitary and quarantine treatment programs, contact and deal preparation and sanitary disinfection units, site supervision and evaluation of treatment effect, issuing certificates and other processing by the media department of biological monitoring and control regulation, sanitary and quarantine treatment increased the intensity of supervision and scientific, will further standardize sanitary disinfection work. Since the inspection and quarantine departments clear responsibility and authority for sanitary disinfection units, strengthening pre-treatment, the whole process of regulatory treatment, after treatment, will promote the standardized operation of sanitary and quarantine treatment units to reduce the "stealing two pounds short." and respond to perfunctory phenomenon, the effect of improving sanitary and quarantine treatment, and reduce the occurrence of safety accidents.

Contributing to the development of the Port of vector background investigation work, and promote the development of culture laboratories and professional and technical personnel. Since the vector surveillance and control work is relatively independent, relatively fixed staff, media port Infectious biological control programs, monitoring programs and specific objectives planned in order to better develop, port medium term planning biological background of the investigation was possible; "and personnel", "given method," "given time" and other technical requirements could truly implement; port infectious Disease vectors population density, distribution and other investigations can truly orderly conduct. Meanwhile, the port and the background investigation conducted media monitoring of bio-dynamic media for biological identification and detection laboratory construction and development provides an opportunity for the laboratory standardized management and on the scale, level to provide a service guarantee.

\section{Conclusion}

Medical vector is a class of creatures serious harm to human health, can spread a variety of diseases. Methods and measures to take scientific, accurate and effective medical biological media monitoring, identification and control, so as to establish a sanitary barrier border crossing to prevent by medical vector-borne diseases frontier port inbound and outbound.

\section{REFERENCE}

[1] Zhiping, Pan View. Vectors and international traffic [J]. Chinese Journal of Hygienic Insecticides \& Equipments, 2004, 10 (2): 73-75

[2] Lijun Cheng, Nie Weizhong, Li listen. Influence of foreign objects on health and the environment [J]. Chinese border Health Journal quarantine, 2003,26 (9): 95-99

[3] Nai, the Wang Longwen, Shizong Wei. Remote Authentication Practices and Chinese door Times [N]., 2002-02-27

[4] Wang Hui multimedia and networking applications in the TV monitor in the [J]. Journal of Anhui University, 2002, 26 (1): 24-29

[5] Zhang Rongming. Internet Remote Monitoring System Design [J]. Tianjin-based industrial University., 2007 